\title{
Parenchyma-sparing vessel-guided major hepatectomies: Nonsense or new paradigm?
}

\author{
Guido TORZILLI* $^{*}$
}

Humanitas University, Italy

Lecture: Liver surgery has seen several developments to increase resectability and maintain safety [1]. Among the most relevant techniques are portal vein embolization, two stage hepatectomy, associated liver partition and portal vein ligation for staged hepatectomy (ALPPS), and liver venous deprivation followed by major hepatectomy. While these solutions are designed to boost the future liver remnant, they all pursue a major vessel amputation. Even if such major liver surgery is done to increase resectability, the removal of large parts of the organ may reduce the chance of redo surgery for recurrent disease when compared to parenchyma-sparing alternatives. However, developments in extreme parenchyma-sparing techniques have received less attention over very extensive liver resection. For liver lesions, looking at the target from different viewpoints may offer different perspective to solutions. One such viewpoint is to challenge the universal pursuit for radical resection (defined as microscopic R0 on pathology), at least in some selected situations. As an example, over half of all liver surgeons would accept tumor exposure (R1) by necessity when doing surgery for colorectal liver metastases [2]. For hepatocellular carcinoma (HCC), the adequate surgical margin is still debated with competition between the issue of radicality and preservation of liver function. For mass-forming cholangiocarcinoma however, most surgeons argue for a clear surgical margin (e.g. $>1 \mathrm{~cm}$ ) even though an independent role of the margin per se has not been confirmed across several reports [3].

One particular setting in which tumor exposure could potentially represent an acceptable oncological approach to resection is the situation when there is tumor-vessel contact. Major intrahepatic vessels are anatomical boundaries separating totally distinguished liver portions. Furthermore, major intrahepatic vessels are wrapped by the Leannec capsule in addition to the Glissonean sheat, or vein wall [4]. Then, the Laennec capsule is a further barrier between the tumor and the intrahepatic vessel. Given all of that, detaching the tumor from the intrahepatic vessel (referred to as ' $R 1$ vasc') could be at least not heretic. A comparable recurrence risk of R1vasc and R0 surgery for HCC [5], and colorectal liver metastases [6] would confirm the hypothesis in theory. In contrast to these tumors, a mass-forming cholangiocarcinoma [7] should only be considered for a R1vasc when the tumor would otherwise be rendered unresectable.

Sometimes the major vessels cannot be spared because of a lesion infiltrating the vessel. In the event the infiltrated major vessel would be a hepatic vein, the option of major parenchymal removal should not be considered as inevitable. Studies have shown that grafting an infiltrated hepatic vein to spare parenchyma and expand the FLR is feasible but with high morbidity and mortality [8]. However, in presence of tumor-vessel contact not amenable of detachment, a compensatory circulation between the adjacent hepatic veins could be detected almost universally [9]. These communicating veins should be overlooked at preoperative imaging, confirmed at intraoperative ultrasound (IOUS), and preserved during dissection to enhance the chance of parenchyma-sparing liver resection. Indeed, the Rlvasc surgery and the hepatectomies driven by the presence of communicating veins represent the scaffolds of a new modality of liver resection namely the vessel-guided hepatectomy.

A perfect and constant orientation of the liver anatomy by the surgeon during dissection is a prerequisite to pursue tumor-vessel contact as a resectable disease, to profit of the eventual presence of the communicating veins, and at the end to avoid vessel and tissue amputation. To that end at least two technical clues are required. One is expertise in IOUS, and the second is a perfect knowledge of the liver anatomy at hand. To achieve this, the IOUS constitutes the 'eyes' and the major vessels the 'path' through which the vessel-sparing approach may be facilitated. The combination of techniques are required to achieve a roadmap for liver resection towards a parenchyma-sparing attitude. In doing this, the liver surgeon is not just challenging by the anatomical complexity of the liver, but more so 
being guided exactly by its very nature.

Profiting of the complexity of liver anatomy implemented the surgical options, while preserving the liver scaffold increased the salveagiability in case of relapse $[10,11]$. Sculpturing, rather than just amputate the organ also pushes for reconsidering the concept of minor and major hepatectomy [12], implementing if not rewriting the dictionary of liver surgery. A safer clinical outcome after major tissue deprivation in a vessel-guided hepatectomy fashion compared to that following major resections through conventional vessels amputation should also deserve some consideration $[13,14]$. ALPPS has shown to be associated with an increment in liver volume which does not translate one to one with liver function [15]. Vessel-guided hepatectomy keeping the organ scaffold even after removal of larger amount of liver tissue, as it happens in case of multiple complex resection for bilobar colorectal liver metastases, has shown a low rate of liver failure [13,14]. Milder regeneration of the liver after vessel-guided hepatectomy compared to that evident after major amputation of the organ may not be just a suggestion. Possibly, vessel-guided hepatectomy let foresee a more tolerable large tissue deprivation: the paradox of parenchymal sparing major hepatectomies (Fig. 1).

Despite all the strengths and opportunities for improving liver resection by vessel-guided resection, the implementation into a wider clinical practice remains to be demonstrated. As a technically sophisticated procedure, vessel-guided hepatectomy is first-and-foremost based on the surgeon-skill resources rather than on sophisticated and expensive technology-all that is needed is basically an ultrasound probe, a Kelly clamp, and a pair of Metzenbaum scissor. The procedure cost is comparably low but the clinical applicability potentially wide. Indeed, vessel-guided hepatectomy would be potentially suitable also in those areas with a health system suffering economical restrictions. However, the share absence of any highly technological equipment makes the interest from the health industry low, and a reduced attention and fewer sponsored opportunities for training and workshops accordingly. Comparably, the partial applicability of vessel-sparing resections to the concept of minimal invasive liver surgery that currently attracts large investments of the health industry stands in stark contrast to the visibility within the surgical community of robotics and laparoscopic techniques. Multiplanar dissections result difficult in minimal access surgery while staged procedures do not: for sure this fact does not represent an incentive for the spread of vessel-guided hepatectomy [16]. Furthermore, the vessel-guided hepatectomy technique is a modality requiring long-term training for which tutorship is currently lacking. However, in author's own experience, a young surgical team (mean age of 36 years) could independently cover up to $80 \%$ of surgical procedure carried out on a yearly basis within 5-6 years of the learning curve [17].

On a final note, the 1980s saw Makuuchi et al. [18] introduce the anatomical parenchyma sparing hepatectomy for patients normally operated with risky major resections. Then, in the 1990s Bismuth et al. [19] introduced the conversion chemotherapy to 'resect the unresectable'. In the 2020s, the vessel-guided hepatectomy has extended parenchymal sparing hepatectomy into the territory of major

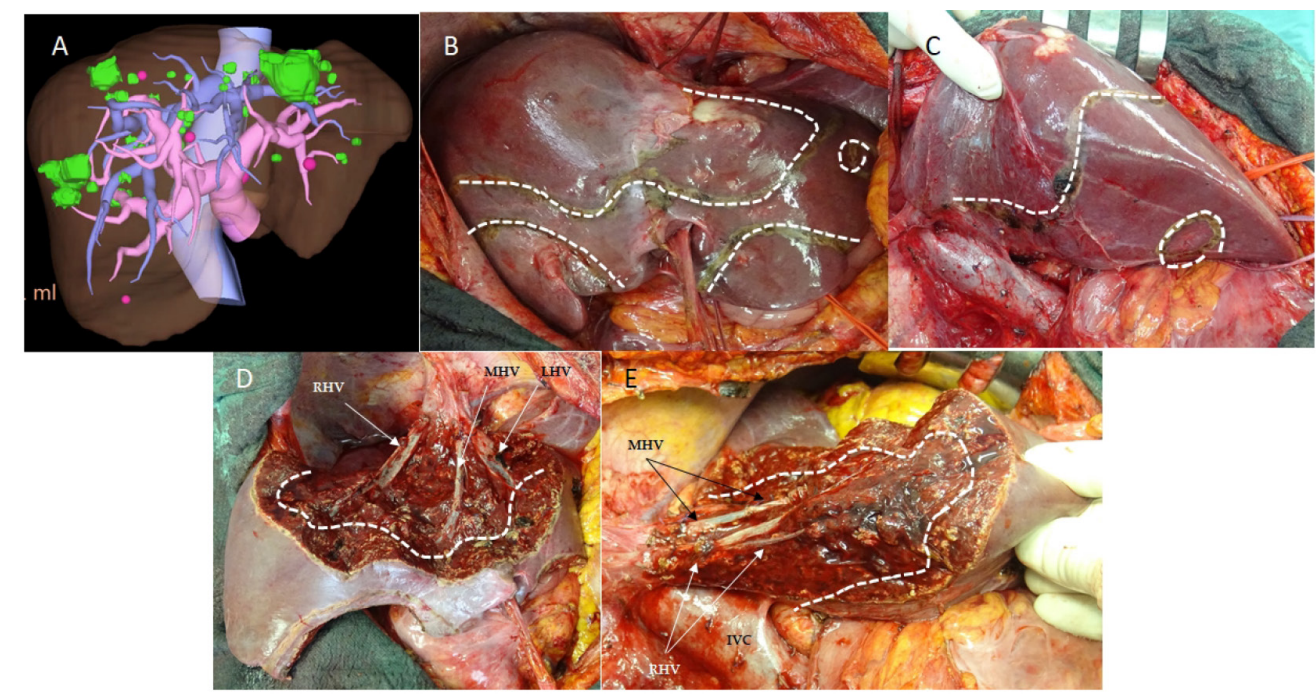

Fig. 1. (A) Virtual cast of a patients carrier multiple (17) cysts (violet) and multiple (36) colorectal liver metastases (green); (B) the virtual cast with the planned resections areas (solid portions); (C) the virtual cast with the planned FLR (solid portion) for a total of $42 \%$ of the total liver volume; (D-F) resection areas marked by yellow dotted lines; (G-I) cut surfaces with the green dotted lines indicating Rlvasc resection and the yellow dotted line showing a tunnel between the paracaval portion of the left hepatic vein (LHV), the segment 1 cut surface, and the inferior vena cava (IVC). GP8v1 and GPv2, glissonean pedicles to subsegment 8 ventral; MHV, middle heaptic vein; RHV, right hepatic vein; UP, umbilical portion. 
hepatectomy and beyond - again, to 'resect the unresectable' but this time based on reintroduction of a new surgical paradigm. Only the future will tell if vessel-guided hepatectomy conveying the paradox combination of parenchyma-sparing and major hepatectomy is a step forward or not.

Key-words: R1vasc; Intraoperative ultrasound; Cholangiocarcinoma; HCC; Colorectal liver metastases; ALPPS; Parenchyma sparing hepatectomy: two stage hepatectomy

\section{CONFLICT OF INTEREST}

No potential conflict of interest relevant to this article was reported.

\section{REFERENCES}

1. Wigmore SJ. Advances in liver surgery. Br J Surg 2020;107:788-789.

2. Viganò L, Costa G, Cimino MM, Procopio F, Donadon M, Del Fabbro D, et al. R1 resection for colorectal liver metastases: a survey questioning surgeons about its incidence, clinical impact, and management. J Gastrointest Surg 2018;22:1752-1763.

3. Mavros MN, Economopoulos KP, Alexiou VG, Pawlik TM. Treatment and prognosis for patients with intrahepatic cholangiocarcinoma: systematic review and meta-analysis. JAMA Surg 2014;149:565-574.

4. Sugioka A, Kato Y, Tanahashi Y. Systematic extrahepatic Glissonean pedicle isolation for anatomical liver resection based on Laennec's capsule: proposal of a novel comprehensive surgical anatomy of the liver. J Hepatobiliary Pancreat Sci 2017;24:17-23.

5. Donadon M, Terrone A, Procopio F, Cimino M, Palmisano A, Viganò L, et al. Is R1 vascular hepatectomy for hepatocellular carcinoma oncologically adequate? Analysis of 327 consecutive patients. Surgery 2019;165:897-904.

6. Viganò L, Procopio F, Cimino MM, Donadon M, Gatti A, Costa G, et al. Is Tumor detachment from vascular structures equivalent to R0 resection in surgery for colorectal liver metastases? An observational cohort. Ann Surg Oncol 2016;23:1352-1360.

7. Torzilli G, Viganò L, Fontana A, Procopio F, Terrone A, Cimino MM, et al. Oncological outcome of R1 vascular margin for mass-forming cholangiocarcinoma. A single center observational cohort analysis. HPB (Oxford) 2020;22:570-577.

8. Hemming AW, Reed AI, Langham MR, Fujita S, van der Werf WJ, Howard RJ. Hepatic vein reconstruction for resection of hepatic tumors. Ann Surg 2002;235:850-858.

9. Torzilli G, Garancini M, Donadon M, Cimino M, Procopio F, Montorsi M. Intraoperative ultrasonographic detection of communicating veins between adjacent hepatic veins during hepatectomy for tumours at the hepatocaval confluence. Br J Surg 2010;97:18671873.

10. Torzilli G, Viganò L, Gatti A, Costa G, Cimino M, Procopio F, et al. Twelve-year experience of "radical but conservative” liver surgery for colorectal metastases: impact on surgical practice and oncologic efficacy. HPB (Oxford) 2017;19:775-784.

11. Torzilli G, Procopio F, Viganò L, Costa G, Fontana A, Cimino M, et al. The liver tunnel: intention-to-treat validation of a new type of hepatectomy. Ann Surg 2019;269:331-336.

12. Viganò L, Torzilli G, Troisi R, Aldrighetti L, Ferrero A, Majno P, et al.; CLISCO group. Minor hepatectomies: focusing a blurred picture: analysis of the outcome of 4471 open resections in patients without cirrhosis. Ann Surg 2019;270:842-851.

13. Torzilli G, Viganò L, Cimino M, Imai K, Vibert E, Donadon M, et al. Is enhanced one-stage hepatectomy a safe and feasible alternative to the two-stage hepatectomy in the setting of multiple bilobar colorectal liver metastases? A comparative analysis between two pioneering centers. Dig Surg 2018;35:323-332.

14. Torzilli G, Serenari M, Viganò L, Cimino M, Benini C, Massani M, et al. Outcomes of enhanced one-stage ultrasound-guided hepatectomy for bilobar colorectal liver metastases compared to those of ALPPS: a multicenter case-match analysis. HPB (Oxford) 2019;21:1411-1418.

15. Olthof PB, Tomassini F, Huespe PE, Truant S, Pruvot FR, Troisi RI, et al. Hepatobiliary scintigraphy to evaluate liver function in associating liver partition and portal vein ligation for staged hepatectomy: liver volume overestimates liver function. Surgery 2017;162:775-783.

16. Melandro F, Giovanardi F, Hassan R, Larghi Laureiro Z, Ferri F, Rossi M, et al. Minimally invasive approach in the setting of ALPPS procedure: a systematic review of the literature. J Gastrointest Surg 2019;23:1917-1924.

17. Torzilli G, McCormack L, Pawlik T. Parenchyma-sparing liver resections. Int J Surg 2020;82S:192-197.

18. Makuuchi M, Hasegawa H, Yamazaki S. Ultrasonically guided subsegmentectomy. Surg Gynecol Obstet 1985;161:346-350.

19. Bismuth H, Adam R, Lévi F, Farabos C, Waechter F, Castaing D, et al. Resection of nonresectable liver metastases from colorectal cancer after neoadjuvant chemotherapy. Ann Surg 1996;224:509-520; discussion 520-522. 